\title{
A comprehensive study of the effect of reactive end groups on the charge carrier transport within polymerized and nonpolymerized liquid crystals
}

\author{
R. J. Baldwin and T. Kreouzis ${ }^{\text {a) }}$ \\ Physics Department, Queen Mary, University of London, Mile End Road London E1 4NS, \\ United Kingdom \\ M. Shkunov, M. Heeney, W. Zhang, and I. McCulloch \\ Merck Chemicals, Chilworth Science Park, Southampton SO16 7QD, United Kingdom
}

(Received 11 May 2006; accepted 20 November 2006; published online 30 January 2007)

\begin{abstract}
Polymerizable liquid crystalline semiconductors, referred to as reactive mesogens (RMs), consist of $\pi$-conjugated cores with reactive end groups decoupled by an aliphatic spacer. These can be polymerized within the mesophase, maintaining the self-assembled morphology and charge transport characteristics. The polymerized films can then be used in organic electronic applications such as charge transport layers in organic light emitting diodes and field effect transistors. We present a systematic study of the effect of reactive end groups on charge transport in calamitic liquid crystals (RMs) using the time-of-flight technique. Several different compounds were synthesized with a variation in both the liquid crystal (LC) mesogenic core group and the functional end groups. The reactive end groups in most cases affect the mesophase charge transport compared to the nonreactive LC mesophase transport. This manifests itself as a reduction in mobility, varying from a factor of 4 in the best case to as large as two orders of magnitude. In the best systems studied, however, the reactive end group effect on the transport, compared to the nonreactive mesophase transport, is negligible. Polymerized reactive mesogens do maintain long-range transport, with comparable mobilities to those of the phase in which they were polymerized over a broad temperature range, including room temperature. The hole and electron mobilities found in polymerized systems are explored using the Holstein small polaron model in the nonadiabatic limit, yielding the relevant polaron binding energies and bandwidths, and using the Bässler Gaussian disorder model, yielding the relevant energetic disorder parameters. (c) 2007 American Institute of Physics. [DOI: 10.1063/1.2432045]
\end{abstract}

\section{INTRODUCTION}

In recent years the interest in organic semiconductors has grown, mainly due to the success of amorphous thin films in devices such as organic light emitting diodes (OLEDS) and Xerographic copiers. Mobilities of charge carriers in such thin films are in the order of $10^{-5}-10^{-3} \mathrm{~cm}^{-2} \mathrm{~V}^{-1} \mathrm{~s}^{-1}$, which limits the performance of organic devices.

Since the discovery of conduction in conjugated calamitic liquid crystals in the $1990 \mathrm{~s},{ }^{1,2}$ many liquid clystals (LCs) have been characterized and found to have charge carrier mobilities in the order of $10^{-4}-10^{-1} \mathrm{~cm}^{-2} \mathrm{~V}^{-1} \mathrm{~s}^{-1}$, thus having superior charge carrier mobilities compared to most amorphous thin films, making them good candidates for semiconductor devices.

Charge transport, however, is fastest in highly ordered liquid crystal mesophases, which usually occur above room temperature, and in many cases when cooled to room temperature these form polycrystalline solids, inducing grain boundaries and other defects which in turn result in trapping, causing unfavorable charge transport properties.

A possible solution is to incorporate polymerizable end

${ }^{a)}$ Electronic mail: t.kreouzis@qmul.ac.uk groups into the molecular structure of the conjugated liquid crystals forming reactive mesogens (RMs). ${ }^{3}$ As with nonreactive mesogens, they are able to form mesophases, which once formed, can be polymerized, and in doing so produce an insoluble network, which maintains the structure of the mesophase and hopefully the charge transport properties at room temperature. ${ }^{4}$

This process is additionally advantageous in the production of multilayer semiconductor devices such as organic field effect transistors (OFETs) and OLEDs for two reasons: (1) sequential solution based application methods can be easily used without the solvent from the most recently applied layer facilitating interlayer diffusion and (2) these devices can be laterally patterned by photolithography.

It has been reported that the incorporation of such polymerizable end groups reduces the charge carrier mobility significantly, when compared to the original nonreactive material, ${ }^{5}$ although, for nematic phase RMs, the mobility appears to increase after the polymerization. ${ }^{6}$ This has been attributed to improvements in positional order and therefore increased $\pi$-orbital overlap.

No major systematic studies of these systems have ever been reported in the literature. Thus in this paper we aim to discuss the effect these reactive end groups have on the charge carrier transport properties. We report both RM sys- 
tems with transport that compares unfavorably with nonreactive LC systems as well as RM systems with desirable transport properties.

\section{MATERIALS AND EXPERIMENTAL SETUP}

The general transport behavior observed in calamitic LC systems shows an improvement in mobility as the material forms higher order mesophases. The higher the order of the mesophase, the better the $\pi$-orbital overlap between neighboring molecules and the higher the subsequent carrier mobility. ${ }^{1,7}$ Consequently one expects higher mobilities in, for example, a smectic $G$ phase compared to a nematic phase.

Three mesogenic cores were used in this study. The first set of materials was based on substituted phenylnaphthalenes (PNP). These were chosen due to the well-known behavior of the original nonreactive liquid crystal, which exhibited ambipolar transport with high mobilities, studied by Funahashi and Hanna. ${ }^{1}$ The second set of materials was based on substituted bis(4-heptylphenyl)-bithiophene (PTTP), which has previously been reported to demonstrate high field effect mobilities as a polycrystalline film. ${ }^{8}$ The third and final set of materials was based on substituted quaterthiophenes (QT). Quaterthiophenes were chosen as they had been shown to have excellent field effect mobilities as vacuum deposited thin films, ${ }^{9}$ and demonstrate liquid crystalline behavior when substituted with alkyl chains. ${ }^{5}$ Compounds $1 \mathrm{~b}, 1 \mathrm{c}$, and $10 \mathrm{~b}$ were prepared as previously reported. ${ }^{4}$ The details of the preparation of the other materials can be found in Ref. 10 .

Each of the mesogenic cores was substituted with various functional end groups, namely, acrylate, diene, and oxetane. All end groups could be polymerized in the presence of a suitable initiator; free radical initiators were used for the acrylate and diene end groups, and a cationic initiator was used for the oxetane group. The use of common conjugated cores allowed the study of how each of the end groups affected the charge transport properties of the core LC group, both before and after polymerization.

The phase behavior of all the materials was characterized using differential scanning calorimetry (DSC) and polarized microscopy. Thermal transitions were determined by using a Perkin Elmer DSC-7. Liquid crystalline textures were observed under a Leica DMLP optical microscope with a Linkam DMLP hot stage and TMS93 controller.

Glass liquid crystal cells with indium tin oxide (ITO) electrodes, with known interelectrode distances, were mounted on a modified Linkam LTS350 hot stage. The cells used varied in thickness from $\sim 1$ to $\sim 10 \mu \mathrm{m}$ and were coated with antiparallel rubbed polyimide alignment layers. The liquid crystal cell was filled by capillary action by melting the material above the clearing point and then cooled to the required mesophases. Connected to one of the electrodes is the output from a variable dc power supply, allowing the electric field to be varied. The counterelectrode is connected via a load resistor to the ground at the input of an Agilent infinium digitizing oscilloscope.

A 6 ns UV (337 nm) pulse from a Lambda Physik $\mathrm{N}_{2}$ laser incident upon the top electrode is used to create electron-hole pairs within a skin depth $(\delta \approx 100 \mathrm{~nm})$. In the case of the top electrode being positive, electron recombination occurs at the top electrode, while the holes drift through the bulk of the sample, inducing a current in the external circuit, and before recombining at the counterelectrode. For electron transport the direction of the electric field is simply reversed. Thus the transit time $t_{t}$ of the photogenerated carriers traversing the liquid crystal was determined from an inflection point on a double logarithmic plot of the transient photocurrent. Charge carrier mobility $\mu$ was then calculated using the equation $\mu=d^{2} / V t_{t}$, or in the case of field independent mobility, by the gradient of a drift velocity versus electric field plot. The signal to noise ratio was improved by signal averaging and the coherent rf noise, generated by the laser, is eliminated by background subtraction.

In order to polymerize the materials the addition of a suitable photoinitiator is needed, typically $0.5 \mathrm{wt} \%$. The photoinitiators Irgacure 651 (2,2-dimethoxy-1,2-diphenylethan-1-one, Ciba Geigy) and Omnicat 550 (10-biphenyl-4yl-2-isopropyl-9-oxo-9H-thioxanthen-10-ium hexafluorphosphate, IGM resins) were used for the polymerization of diene and oxetane end groups, respectively. The sample is then loaded as previously described and cooled to the relevant mesophase. Photopolymerization is achieved using the $405 \mathrm{~nm}$ wavelength output from a continuous wave diode laser $(15 \mathrm{~mW})$ for over $60 \mathrm{~min}$. Although the domain size formed is generally a function of material, mesophase, and cooling rate on formation, the polymerized films showed similar domain sizes to the nonpolymerized samples, typically of the order of $25 \mu \mathrm{m}$ or greater in the smectic phase. The polymerization of the sample was confirmed by raising the sample temperature above the clearing point and, under polarized microscopy, observing the illuminated areas maintain their phase structure, in contrast to the nonilluminated area which underwent transition to the isotropic phase. No phase changes were observed in the polymerized films during the heating procedure. For a full and comprehensive review of polymerization involving RMs please see Ref. 11

\section{RESULTS}

\section{A. Charge carrier transport in PNP materials}

Initial studies were carried out on the set materials with the mesogenic core phenylnaphthalene (see Table I for phase

TABLE I. Phase behavior of liquid crystal and reactive mesogen materials used. a denotes measured or cooling cycle and b denotes measured on heating cycle.

\begin{tabular}{ccc}
\hline \hline Material & Phase behavior (temperatures in $\left.{ }^{\circ} \mathrm{C}\right)$ & \\
\hline 8PNPO12 & K-85-SmB-101-SmA-122-I & $\mathrm{b}$ \\
PNP-acrylate & K-50-SmC-66-SmA-109-I & $\mathrm{a}$ \\
PNP-diene & K-(-10)-SmG-59-SmC-76-I & $\mathrm{a}$ \\
PTTP & K-140-SmG-225-SmC-229-SmA-234-I & $\mathrm{b}$ \\
PTTP-diene & K-75-SmG-174-I & $\mathrm{b}$ \\
PTTP-oxetane & K-83-SmG-93-SmB-144-I & $\mathrm{a}$ \\
QT & K-74-SmG-173-I & $\mathrm{a}$ \\
QT-diene & K-(-20)-SmH-50-SmG-96-I & $\mathrm{a}$ \\
QT-oxetane & K-73-SmB-110-I & $\mathrm{a}$ \\
\hline \hline
\end{tabular}


<smiles>CCCCc1ccc(-c2ccc3cc(OCC)ccc3c2)cc1</smiles>

(a)<smiles>C=CC(=O)OCCCCCCOc1ccc(-c2ccc3cc(OCCCCCCOC(=O)C=C)ccc3c2)cc1</smiles>

(b)

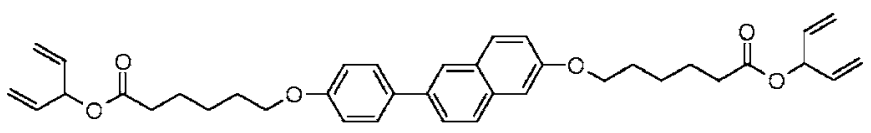

(c)

FIG. 1. Molecular structures of the phenylnaphthalene LCs: (a) nonreactive phenylnaphthalene (8PNPO12), (b) phenylnaphthalene acrylate (PNPacrylate), and (c) phenylnaphthalene diene (PNP-diene).

behavior). Only hole transport was investigated, since the samples were prepared in air, and the presence of oxygen is known to suppress electron transport.

The hole transport observed in the smectic A phase of 8PNPO12 [Fig. 1(a)] was in excellent agreement with literature values reported by Funahashi and Hanna. ${ }^{1}$ The transport was nondispersive and with a mobility of 2.7 $\times 10^{-4} \mathrm{~cm}^{2} \mathrm{~V}^{-1} \mathrm{~s}^{-1}\left(\mathrm{SmA}, 110^{\circ} \mathrm{C}\right)$.

In both phenylnaphthalene RMs, the hole transport in the smectic phase became dispersive and the mobility was significantly reduced. For PNP-acrylate [Fig. 1(b)] the measured hole mobility was $4.9 \times 10^{-5} \mathrm{~cm}^{2} \mathrm{~V}^{-1} \mathrm{~s}^{-1}$ and for PNPdiene [Fig. 1(c)] $7.4 \times 10^{-5} \mathrm{~cm}^{2} \mathrm{~V}^{-1} \mathrm{~s}^{-1}$

Polymerization of PNP-acrylate was then attempted, however, in the presence of photoinitiator the material always thermally polymerized before the cell could be loaded. Consequently, it was concluded that acrylate end groups were too thermally unstable for this processing technique and thus did not allow enough control of phase in which polymerization occurred. In the case of PNP-diene there was a factor of 4 decrease in mobility when compared to the original nonreactive LC, however, we did not investigate this material further (e.g., postpolymerization studies) as higher mobility RMs became available. The PNP family results are summarized in Table II.

\section{B. Charge carrier transport in PTTP materials}

Time-of-flight (TOF) studies were then conducted on the nonreactive PTTP [Fig. 2(a), see Table I for phase behavior]. This material displayed fast ambipolar transport (Fig. 3) in the smectic phase $(\mathrm{SmG})$, with resulting hole mobility $\mu_{\text {hole }}$ $=4.4 \times 10^{-2} \mathrm{~cm}^{2} \mathrm{~V}^{-1} \mathrm{~s}^{-1}$ and electron mobility $\mu_{\text {electron }}=7.1$ $\times 10^{-2} \mathrm{~cm}^{2} \mathrm{~V}^{-1} \mathrm{~s}^{-1}$ (both are dispersive and field dependent,

TABLE II. Summary of PNP family mobilities.

\begin{tabular}{ccc}
\hline \hline Material & $\mu_{\text {hole }}\left(\mathrm{cm}^{2} \mathrm{~V}^{-1} \mathrm{~s}^{-1}\right)$ & Mesophase \\
\hline 8PNPO12 & $2.7 \times 10^{-4}$ & $\mathrm{SmA}$ \\
PNP-acrylate & $4.9 \times 10^{-5}$ & $\mathrm{SmA}$ \\
PNP-diene & $7.6 \times 10^{-5}$ & $\mathrm{SmC}$ \\
\hline \hline
\end{tabular}<smiles>Cc1ccc(-c2ccc(-c3ccc(-c4ccc(C)cc4)s3)s2)cc1</smiles>

(a)

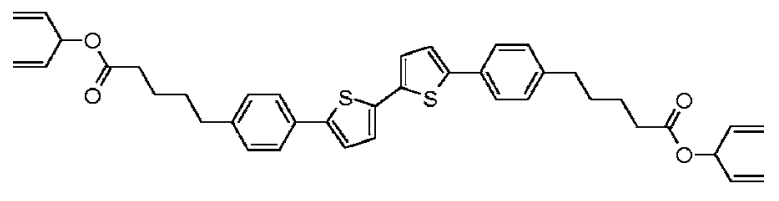

(b)

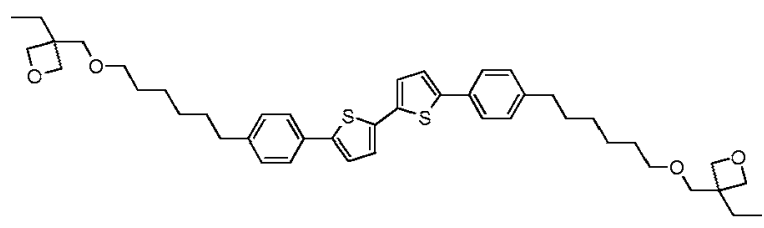

(c)

FIG. 2. Molecular structure of bis(4-heptylphenyl)-bithiophene LCs: (a) nonreactive bis(4-heptylphenyl)-bithiophene (PTTP), (b) bis(4heptylphenyl)-bithiophene diene (PTTP-diene), and (c) bis(4-heptylphenyl)bithiophene oxetane (PTTP-oxetane).

quoted here at $4 \times 10^{4} \mathrm{~V} \mathrm{~cm}^{-1}$ ). These promising LC charge transport properties made this a suitable core for substitution with reactive end groups.

The RM PTTP-diene (nonpolymerized) [Fig. 2(b)] was investigated; this also displayed ambpolar transport, once again in the SmG phase. Both holes and electrons were found to be dispersive. Mobilities were reduced compared to the original material with a drop of almost an order of magnitude for the holes, $\mu_{\text {hole }}=6.6 \times 10^{-3} \mathrm{~cm}^{2} \mathrm{~V}^{-1} \mathrm{~s}^{-1}$, and by a factor of 40 mobility in the electrons, $\mu_{\text {electron }}=1.7$ $\times 10^{-4} \mathrm{~cm}^{2} \mathrm{~V}^{-1} \mathrm{~s}^{-1}$.

The nonpolymerized PTTP-oxetane [Fig. 2(c)] results were much more encouraging. When the material was in the smectic B phase, the electron transport was dispersive, how-

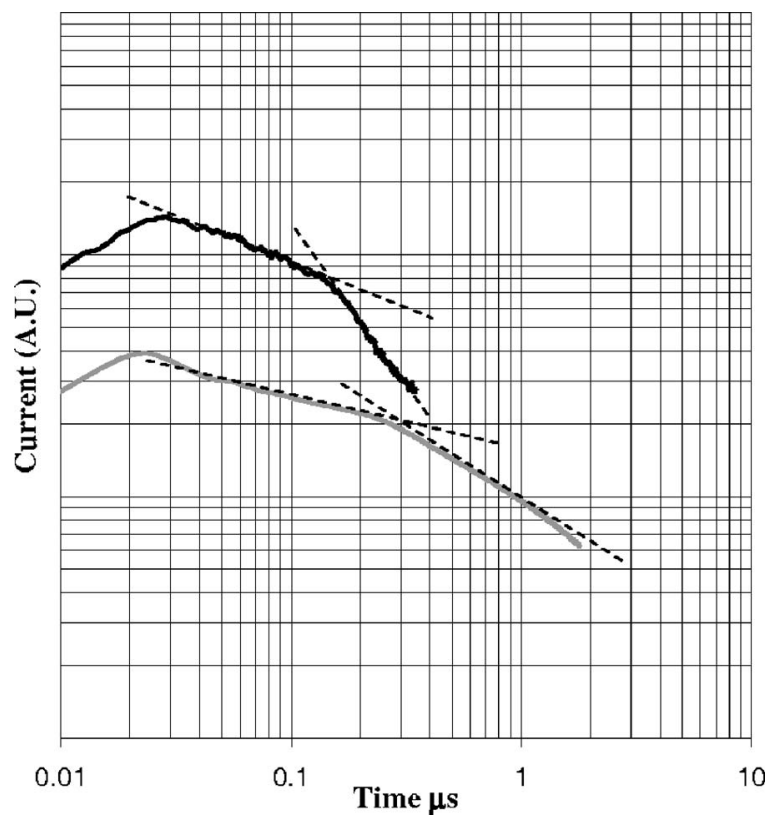

FIG. 3. Electron (upper curve) and hole (lower curve) photocurrents across a $4.9 \mu \mathrm{m}$ sample of PTTP in the smectic phase $\left(E=4 \times 10^{4} \mathrm{~V} \mathrm{~cm}^{-1}\right)$. 


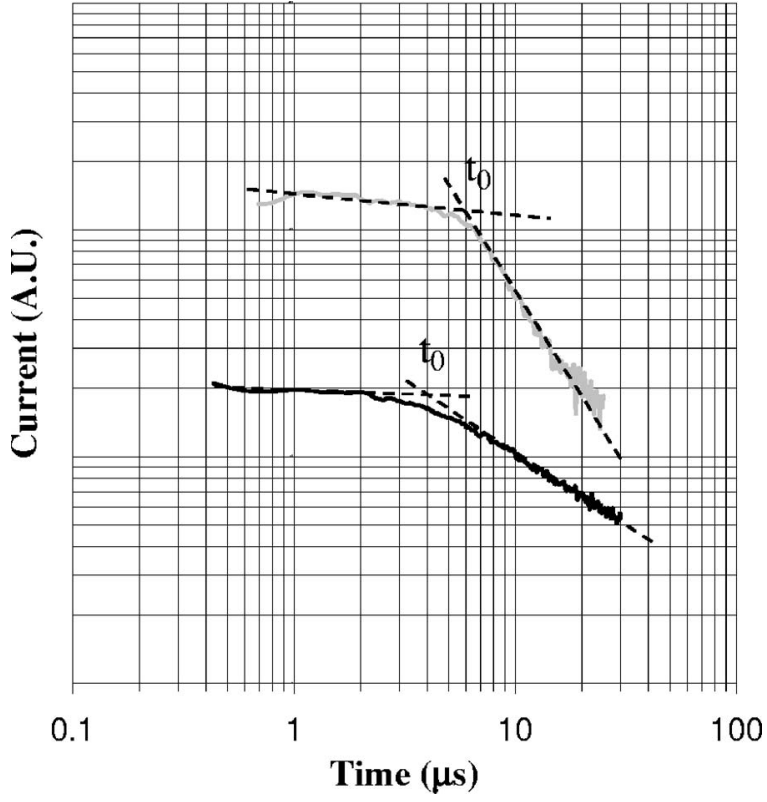

FIG. 4. Hole (upper curve) and electron (lower curve) photocurrents across a $10 \mu \mathrm{m}$ sample of nonpolymerized PTTP-oxetane at $140{ }^{\circ} \mathrm{C}$ (smectic B phase, $20 \mathrm{~V}$ bias).

ever, the mobility measured was much higher than that of PTTP-diene, $\mu_{\text {electron }}=1.7 \times 10^{-4} \mathrm{~cm}^{2} \mathrm{~V}^{-1} \mathrm{~s}^{-1}$. In the $\mathrm{SmB}$ phase the hole transport remained nondispersive, reaching $\mu_{\text {hole }}=9.5 \times 10^{-3} \mathrm{~cm}^{2} \mathrm{~V}^{-1} \mathrm{~s}^{-1}$ (see Fig. 4). Within the smectic $\mathrm{B}$ phase both holes and electrons displayed transport that was field independent and temperature dependent (Fig. 5). Upon transition into the crystalline phase long featureless decays were observed, implying that trapping was occurring. Thus no mobility could be measured.

PTTP-diene was mixed with 0.5 wt $\%$ initiator for polymerization. The material was then polymerized at $135^{\circ} \mathrm{C}$ (smectic $\mathrm{G}$ ). The charge transport was measured from 15 to $195^{\circ} \mathrm{C}$, electron and hole mobilities appear to be both independent of temperature and electric field (Fig. 6), typically $\mu_{\text {electron }}=7.5 \times 10^{-4} \mathrm{~cm}^{2} \mathrm{~V}^{-1} \mathrm{~s}^{-1}$ and $\mu_{\text {hole }}=7.1$ $\times 10^{-4} \mathrm{~cm}^{2} \mathrm{~V}^{-1} \mathrm{~s}^{-1}$. Electron transport was found to be dispersive, while hole transport was nondispersive.

Polymerization of PTTP-oxetane was carried out as previously described this time using $0.5 \mathrm{wt} \%$ cationic initiator.

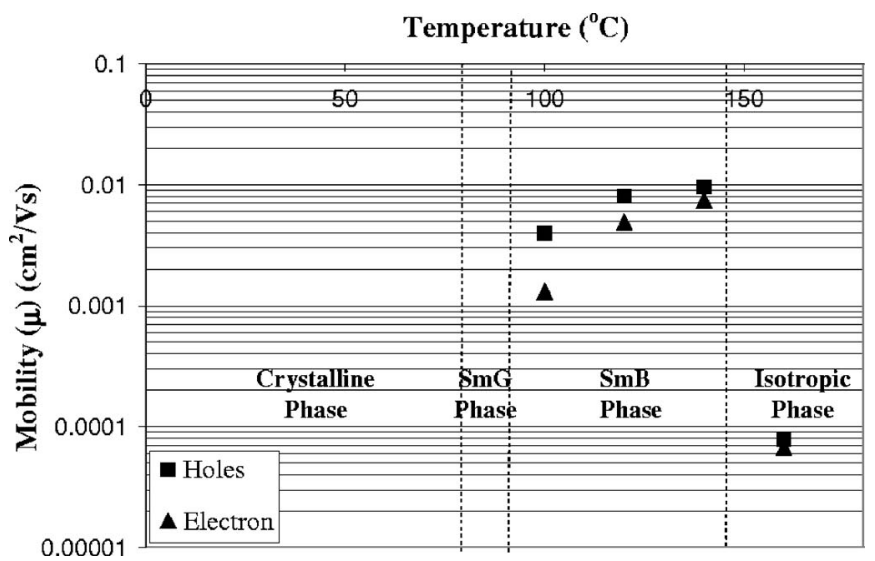

FIG. 5. Mobilities as a function of temperature for PTTP-oxetane (nonpolymerized).

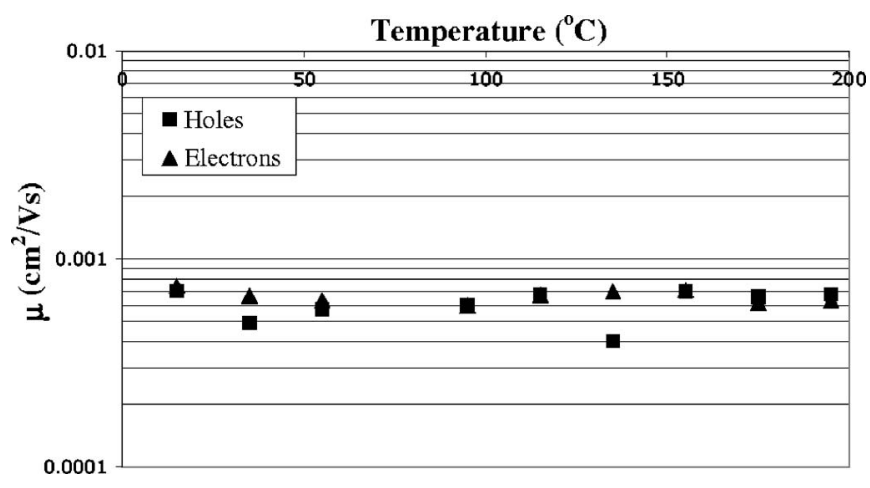

FIG. 6. Mobilities as a function of temperature for PTTP-diene (polymerized).

The polymerization process was carried out at $140{ }^{\circ} \mathrm{C}$ $(\mathrm{SmB})$. The sample was then cooled to $15^{\circ} \mathrm{C}$ and a field independent hole mobility was measured, $\mu_{\text {hole }}=1.6$ $\times 10^{-2} \mathrm{~cm}^{2} \mathrm{~V}^{-1} \mathrm{~s}^{-1}$ (Fig. 7). The electron transport was found to be field dependent and dispersive, $\mu_{\text {electron }}=2.8$ $\times 10^{-2} \mathrm{~cm}^{2} \mathrm{~V}^{-1} \mathrm{~s}^{-1}$ at $4 \times 10^{4} \mathrm{~V} \mathrm{~cm}^{-1}$ (Fig. 8). The hole and electron mobilities displayed weak temperature dependence (Fig. 9). All the PTTP family results are summarized in Table III. The charge transport obtained when PTTP-oxetane was polymerized in the smectic phase was in stark contrast to the charge transport behavior observed when a PTTP-oxetane sample was deliberately polymerized in the isotropic phase. In the case of an isotropic phase polymerized film, featureless long time photocurrent decays were obtained at room temperature, where the carrier transit was not observed.

\section{Charge carrier transport in QT materials}

The third set of materials studied is based on the mesogenic core quaterthiophene (see Table I for phase behavior). Again TOF studies were first conducted on the nonreactive material, QT [Fig. 10(a)]. In the SmG phase the hole

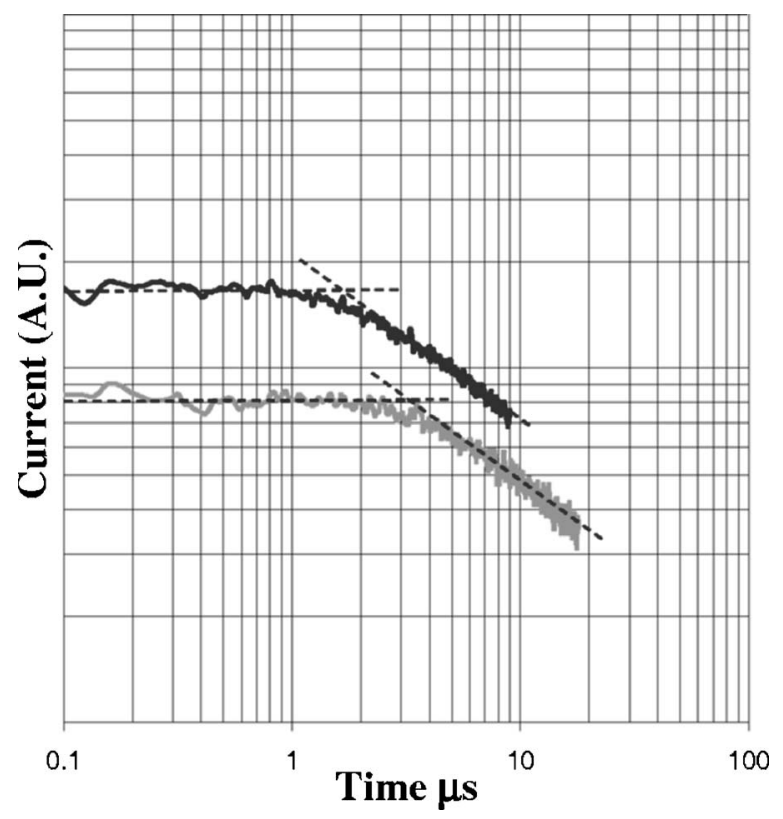

FIG. 7. Hole photocurrent transients across a $10 \mu \mathrm{m}$ film of polymerized PTTP-oxetane (40 V bias upper curve and $20 \mathrm{~V}$ bias lower curve) at $15^{\circ} \mathrm{C}$. 


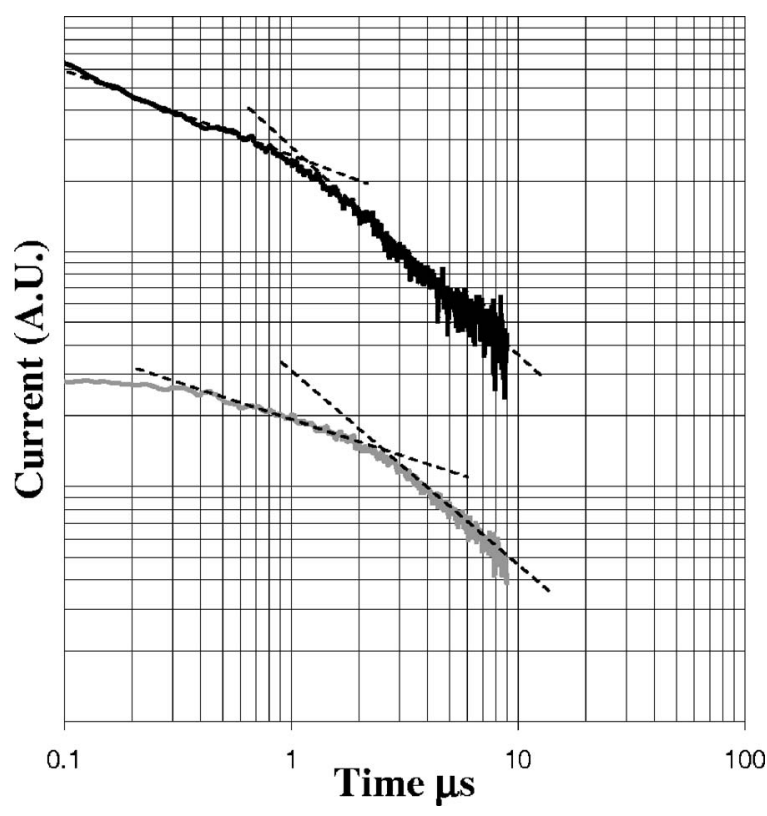

FIG. 8. Electron photocurrent transients across a $10 \mu \mathrm{m}$ film of cross-linked PTTP-oxetane (40 V bias upper curve and $25 \mathrm{~V}$ bias lower curve) at $15{ }^{\circ} \mathrm{C}$.

and electron mobilities were measured to be $\mu_{\text {hole }}=6.3$ $\times 10^{-2} \mathrm{~cm}^{2} \mathrm{~V}^{-1} \mathrm{~s}^{-1}$ and $\mu_{\text {electron }}=7.7 \times 10^{-2} \mathrm{~cm}^{2} \mathrm{~V}^{-1} \mathrm{~s}^{-1}$ (see Fig. 11). The hole transport was nondispersive, while the electron transport was dispersive.

The RM quaterthiophene-diene (QT-diene) [Fig. 10(b)] (nonpolymerized) was then studied. In the SmG phase, hole transport remained nondispersive with a measured mobility $\mu_{\text {hole }}=4.6 \times 10^{-3} \mathrm{~cm}^{2} \mathrm{~V}^{-1} \mathrm{~s}^{-1}$ (see Fig. 12). In the same phase electron transport, however, became highly dispersive and no mobility data could be taken. Strong trapping occurred on further cooling to the next smectic phase below $50{ }^{\circ} \mathrm{C}$.

Nonpolymerized quaterthiophene-oxetane (QT-oxetane) [Fig. 10(c)] was investigated. In the SmB phase the highest mobilities measured were $\mu_{\text {hole }}=5.8 \times 10^{-3} \mathrm{~cm}^{2} \mathrm{~V}^{-1} \mathrm{~s}^{-1}(E$ $\left.=8.9 \times 10^{-4} \mathrm{~V} / \mathrm{cm}\right)$ and $\mu_{\text {electron }}=5.4 \times 10^{-3} \mathrm{~cm}^{2} \mathrm{~V}^{-1} \mathrm{~s}^{-1}(E$ $\left.=8.3 \times 10^{-4} \mathrm{~V} / \mathrm{cm}\right)$. Transport for both the holes and electrons was nondispersive and field dependent, however, the transport became more dispersive as the temperature was lowered.

\section{Temperature $\left({ }^{\circ} \mathrm{C}\right)$}

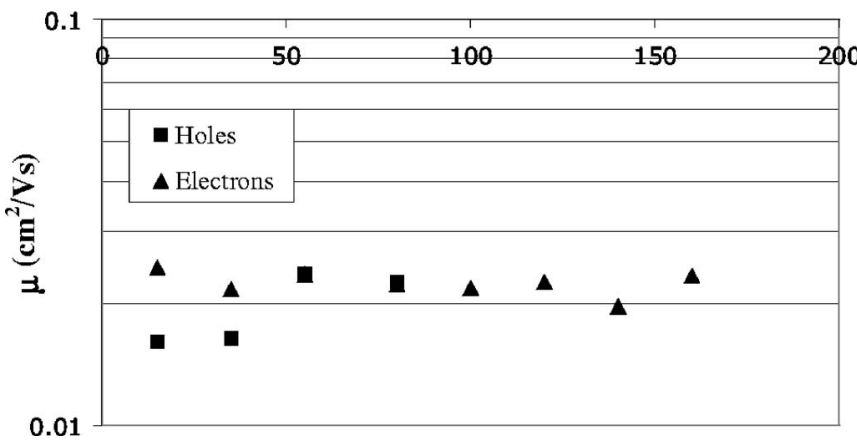

FIG. 9. Mobilities as a function of temperature for PTTP-oxetane (polymerized).

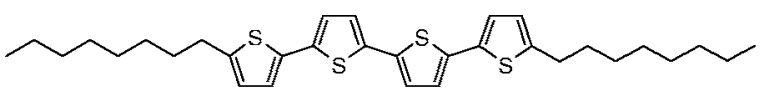

(a)

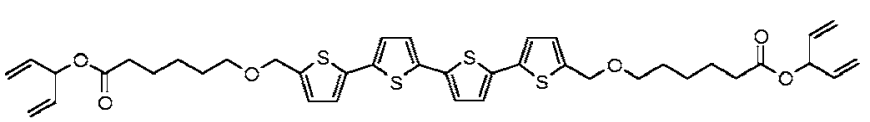

(b)

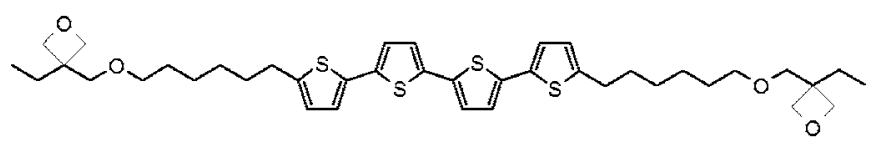

(c)

FIG. 10. Molecular structure of quaterthiophene LCs: (a) nonreactive quaterthiophene (QT), (b) quaterthiophene diene (QT-diene), and (c) quaterthiophene oxetane (QT-oxetane).

A 1 wt $\%$ mixture of photoinitiator with QT-oxetane was then loaded into a $2.45 \mu \mathrm{m}$ cell and cooled to $80{ }^{\circ} \mathrm{C}(\mathrm{SmB})$, where it was polymerized.

The sample was then tested for charge transport properties over the temperature range of $15-140{ }^{\circ} \mathrm{C}$. Typical photocurrent transients for QT-oxetane (polymerized) at $15^{\circ} \mathrm{C}$ are shown in Figs. 13 and 14. The sample exhibited nondispersive ambipolar transport.

Hole and electron mobilities were both temperature and field independent (Fig. 15) and were measured to be $\mu_{\text {hole }}$ $=1.6 \times 10^{-3} \mathrm{~cm}^{2} \mathrm{~V}^{-1} \mathrm{~s}^{-1}$ and $\mu_{\text {electron }}=1.1 \times 10^{-3} \mathrm{~cm}^{2} \mathrm{~V}^{-1}$ $\mathrm{s}^{-1}$.

\section{DISCUSSION}

\section{A. General observations on the charge transport}

Charge transport in reactive mesogens, and their applications to OLEDs have been previously reported in literature. 6,12,13 Hole and electron mobilities for nematic phase calamitic RMs have been low (in the range of $10^{-5}-10^{-3} \mathrm{~cm}^{2} \mathrm{~V}^{-1} \mathrm{~s}^{-1}$ ), much lower than those possible in high order smectic phase liquid crystalline systems. ${ }^{1}$ It is therefore important to begin the molecular design of any cross-linkable system with a LC core, which displays the fastest possible transport.

Table II shows that when the starting material itself (8PNPO12) has low mobility (SmA: $2.7 \times 10^{-4} \mathrm{~cm}^{2} \mathrm{~V}^{-1} \mathrm{~s}^{-1}$ ), any reactive mesogens synthesized will not have exceptional transport properties. We also determined that the thermal instability of the acrylate end group made use of PNP-acrylate difficult, at least when the device processing requires elevated temperatures. Uncontrolled thermal polymerization was not as dramatic in the case of PNP-diene, and this is reflected in the modest improvement of the hole mobility compared to the acrylate, although we cannot rule out that this difference may be due to the size and shape of the diene and the different LC phase. Thermal polymerization introduces an unnecessary complicating factor when attempting to establish the mechanisms affecting the charge transport and is best avoided. Oxetane reactive groups are known to be thermally stable and showed no evidence of thermal crosslinking in any material studied. 
TABLE III. Summary of PTTP family mobilities.

\begin{tabular}{cccc}
\hline \hline Material & $\mu_{\text {hole }}\left(\mathrm{cm}^{2} \mathrm{~V}^{-1} \mathrm{~s}^{-1}\right)$ & $\mu_{\text {electron }}\left(\mathrm{cm}^{2} \mathrm{~V}^{-1} \mathrm{~s}^{-1}\right)$ & Mesophase \\
\hline PTTP & $4.4 \times 10^{-2}\left(\right.$ at $\left.175^{\circ} \mathrm{C}\right)$ & $7.1 \times 10^{-2}\left(\right.$ at $\left.175^{\circ} \mathrm{C}\right)$ & $\mathrm{SmG}$ \\
$\begin{array}{c}\text { PTTP-diene } \\
\text { PTTP-diene }\end{array}$ & $6.6 \times 10^{-3}\left(\right.$ at $\left.140^{\circ} \mathrm{C}\right)$ & $1.7 \times 10^{-4}\left(\right.$ at $\left.140^{\circ} \mathrm{C}\right)$ & $\mathrm{SmG}$ \\
$\begin{array}{c}\text { (polymerized) } \\
\text { PTTP-oxetane }\end{array}$ & $7.1 \times 10^{-4}\left(15^{\circ} \mathrm{C}\right)$ & $7.5 \times 10^{-4}\left(15^{\circ} \mathrm{C}\right)$ & $\ldots$ \\
$\begin{array}{c}\text { (nonpolymerized }) \\
\begin{array}{c}\text { PTTP-oxetane } \\
\text { polymerized })\end{array}\end{array}$ & $9.5 \times 10^{-3}\left(\right.$ at $\left.140^{\circ} \mathrm{C}\right)$ & $7.5 \times 10^{-3}\left(\right.$ at $\left.140^{\circ} \mathrm{C}\right)$ & $\mathrm{SmB}$ \\
\hline \hline
\end{tabular}

The high order SmG phase formed by PTTP yielded excellent electron and hole mobilities, approaching $10^{-1} \mathrm{~cm}^{2} \mathrm{~V}^{-1} \mathrm{~s}^{-1}$ (Table III), making this a suitable candidate LC core. When the transport is compared to the mesophase transport of the two RMs synthesized, PTTP-oxetane and PTTP-diene, the usual reduction in mobility is observed. The PTTP-oxetane results were obtained in the SmB phase and yielded respectable mobilities of the order of $10^{-2} \mathrm{~cm}^{2} \mathrm{~V}^{-1} \mathrm{~s}^{-1}$ for both holes and electrons, corresponding to reductions of factors of $\sim 4$ and $\sim 6$, respectively. In this case, the oxetane end group had a much smaller effect on the transport, compared to the diene, where the mobilities measured in the high order SmG phase were reduced by a factor of $\sim 7$ in the case of holes and a factor of $\sim 1000$ in the case of electrons. The presence of the diene end group clearly affects the electron transport greatly compared to the hole transport. When the hole transport displayed by the two RMs is compared, we note that the higher order SmG phase present in the diene substituted molecules has no compensating effect on the mobility when compared to the lower order SmB phase data obtained from the oxetane substituted molecule. Postpolymerization results yielded long-range charge transport in both systems over a large temperature range, including room temperature, with mobilities comparable to the RM mesophases. In the case of PTTP-oxetane, the elec-

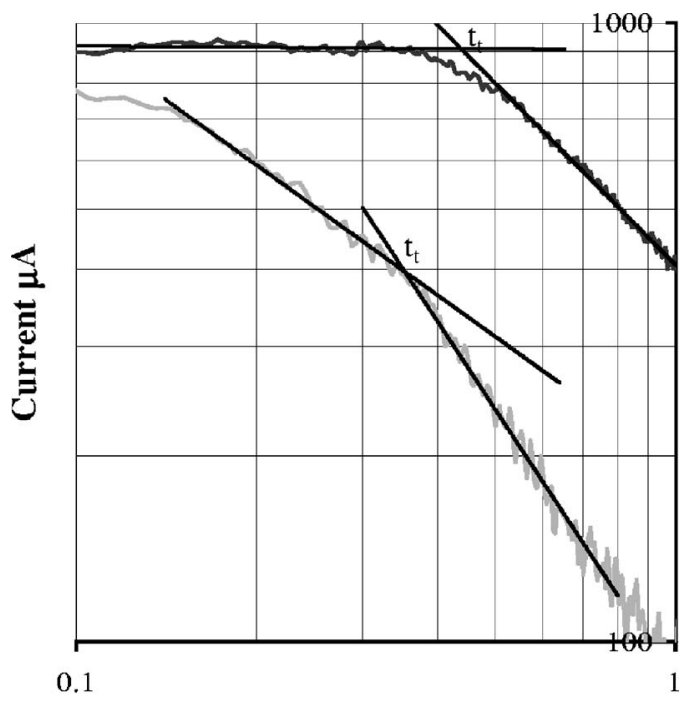

Time $\mu$ s

FIG. 11. Photocurrent transients for holes (upper curve) and electrons (lower curve) in a $4.5 \mu \mathrm{m}$ sample of QT (smectic phase, $7.5 \mathrm{~V}$ bias). tron mobility was found to be temperature independent and the hole mobility showed a weak temperature dependence (Fig. 9), whereas PTTP-diene displayed temperature independent hole and electron mobilities. The electron mobility in PTTP-diene increased postpolymerization, compared to the mesophase value, suggesting that the diene end groups act as chemical traps for the electrons. This is in contrast to the hole data, which showed a decreasing postpolymerization, perhaps as a result of trapping due to the residues from the photoinitiator. If the diene moiety was solely affecting the molecular ordering in the nonpolymerized RM, rather than acting as a chemical trap, we would expect an equally detrimental effect on the charge transport, irrespective of polarity; this is not the case. Although not conclusive, there is indirect evidence for chemical trapping due to the diene moieties. In the case of the polymerized PTTP-oxetane there were modest increases to both electron and hole mobilities compared to the mesophase RM results (it is unclear whether these increases are significant, or simply due to sample to sample variation), however, they approach the values measured for the original LC core. These were previously reported by this group. ${ }^{14}$ The cationic photoinitiator used in the oxetane polymerization reaction did not appear to affect charge transport, in contrast to what is observed from the diene results where a free radical photoinitiator was used.

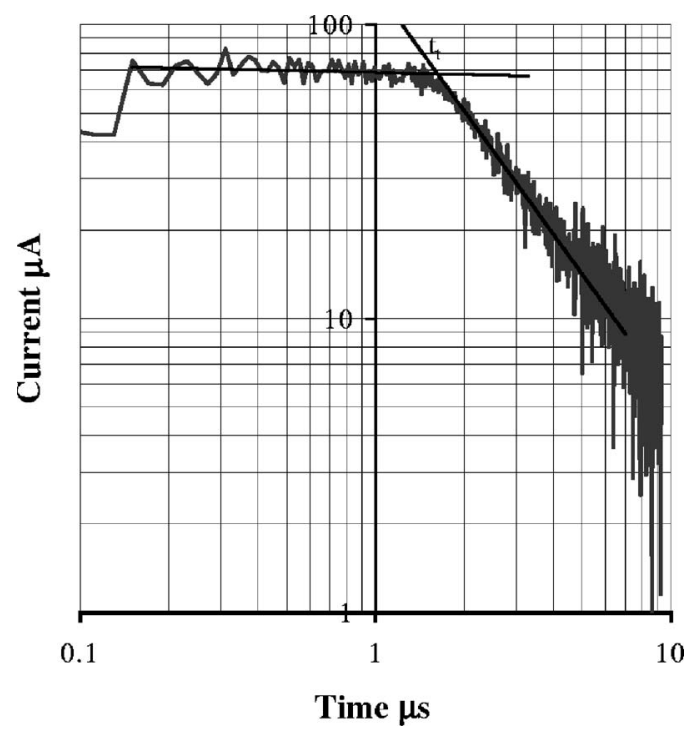

FIG. 12. Photocurrent transient for holes in a $2 \mu \mathrm{m}$ sample of QT-diene (smectic G phase, $27 \mathrm{~V}$ bias) 


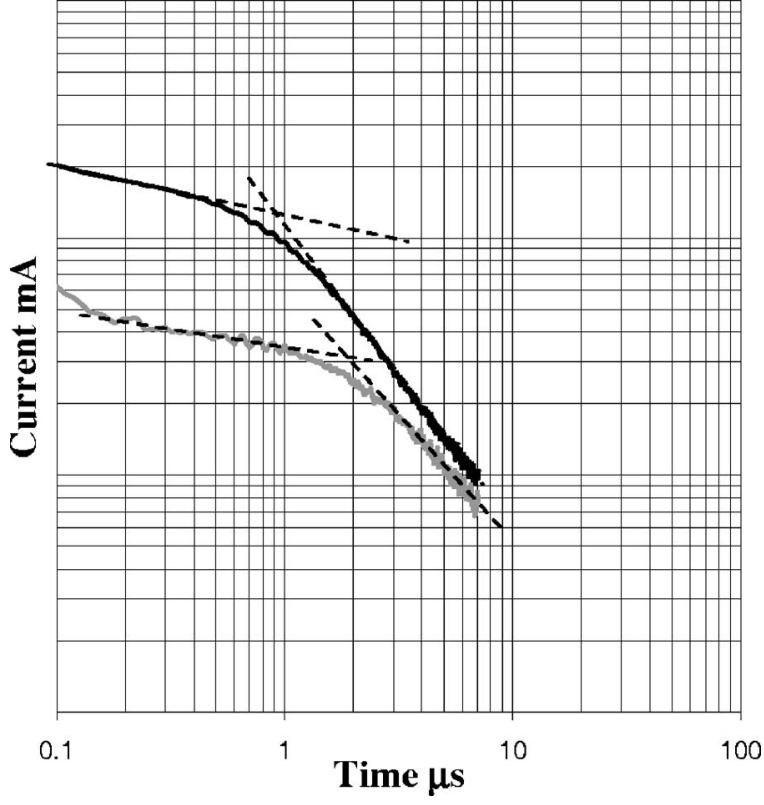

FIG. 13. Hole photocurrent transients across $2.45 \mu \mathrm{m}$ film of polymerized QT-oxetane (41 V bias upper curve and $21 \mathrm{~V}$ bias lower curve).

High electron and hole mobilities, approaching $10^{-1} \mathrm{~cm}^{2} \mathrm{~V}^{-1} \mathrm{~s}^{-1}$, were measured in the SmG phase of QT (Table IV), again suggesting this to be a suitable core for substitution with reactive end groups. The mesophase transport measured in the two RMs shows the relative decrease compared to the nonreactive system, observed in all cases so far. In the case of QT-diene the electron transport was totally suppressed, leading to featureless photocurrent decays, while the hole mobility showed an approximately one order of magnitude drop. The comparison of the mobilities in this case is more straightforward as both LC and RM were measured in the SmG phase. Electron transport was observed in the case of the QT-oxetane RM, although both hole and elec-

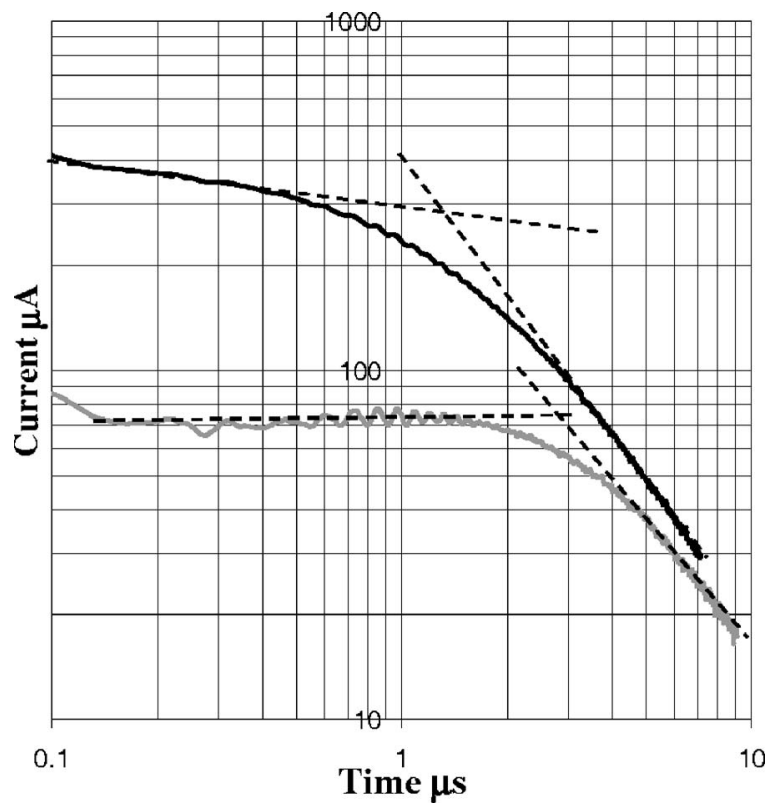

FIG. 14. Electron photocurrent transients across $2.45 \mu \mathrm{m}$ film of polymerized QT-oxetane (41 V bias upper curve and $21 \mathrm{~V}$ bias lower curve). tron mobilities have been reduced by factors of $\sim 9$ and $\sim 11$, respectively, when compared to the LC. When the QToxetane was polymerized there was no recovery of the hole mobility, although the transport was measured over a broad temperature range, remaining largely temperature independent (Fig. 15).

A number of significant observations emerge from this study. Firstly we have demonstrated that the reactive mesogen approach is successful in forming semiconducting layers with desirable transport properties available across a broad temperature range, including room temperature. There is a large variation in these properties, from system to system, however, we can generally observe that diene end groups always reduce electron transport, thus, diene containing RMs are unsuitable where ambipolar transport is needed. We find this not to be the case where the oxetane end group is concerned. Secondly, it is the combination of both core and end groups which is critical in forming high mobility cross-linked films for use in device applications (e.g., PTTPoxetane was successful, whereas QT-oxetane was not). We also find that the choice of photoinitiator used in the polymerization reaction may affect the transport. This, of course, is an example of the well-known effect that impurities have on the transport in all organic systems. ${ }^{15}$ It is also known that morphology plays a crucial role in charge transport, $\mathrm{x}$-ray diffraction studies are being carried out on RM and LC systems in order to investigate how the addition of the end group affects molecular packing and consequently the charge transport properties.

Thirdly, and perhaps most surprisingly, the polymerized films produced display temperature independent charge transport. This is in contrast to amorphous polymer semiconductors where very strong temperature dependence is observed. ${ }^{16,17}$ One could assume that the cross-linked film would behave as a high molecular weight polymer, with corresponding temperature dependent charge transport. Liquid crystalline mesophases commonly display temperature independent transport ${ }^{1,18}$ (albeit within the limited range available within a mesophase) and similar theoretical approaches to those used in LC systems are described in Sec. IV B.

\section{B. Theoretical analysis}

All polymerized RMs described here display temperature independent (or very weakly temperature dependent)

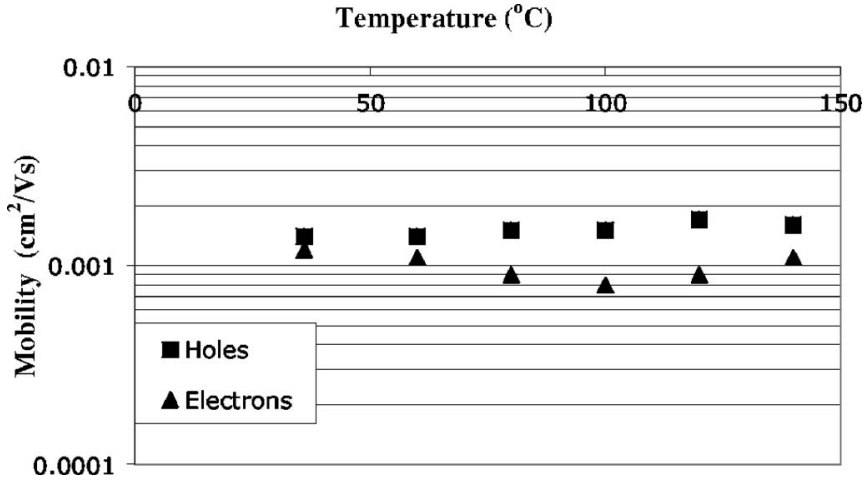

FIG. 15. Mobility-temperature dependence across $2.45 \mu \mathrm{m}$ film of polymerized QT-oxetane. 
TABLE IV. Summary of QT family mobilities.

\begin{tabular}{cccc}
\hline \hline Material & $\mu_{\text {hole }}\left(\mathrm{cm}^{2} \mathrm{~V}^{-1} \mathrm{~s}^{-1}\right)$ & $\mu_{\text {electron }}\left(\mathrm{cm}^{2} \mathrm{~V}^{-1} \mathrm{~s}^{-1}\right)$ & Mesophase \\
\hline QT & $6.3 \times 10^{-2}$ & $7.7 \times 10^{-2}$ & $\mathrm{SmG}$ \\
QT-diene & $4.6 \times 10^{-3}$ & $\cdots$ & $\mathrm{SmG}$ \\
QT-oxetane & $5.8 \times 10^{-3}$ & $5.4 \times 10^{-3}$ & $\mathrm{SmB}$ \\
QT-oxetane & $1.6 \times 10^{-3}$ & $1.1 \times 10^{-3}$ & $\cdots$ \\
(polymerized) & & & \\
$1 \mathrm{wt} \%$ initiator & & & \\
\hline \hline
\end{tabular}

mobilities for both holes and electrons. Traditional hopping models used for organic materials, such as polymers, are usually applied to highly temperature dependent transport. ${ }^{19,20}$ The well-known Bässler disorder formalism, ${ }^{19}$ which considers the motion of the charges to hop within a Gaussian distribution of the energies and distances of the sites, has been used in polymer semiconductor literature, ${ }^{16,17}$ where the hopping mechanism itself is viewed as a thermally activated process of overbarrier jumps and tunneling between hopping sites. Thus hopping mobility is both field and temperature dependent. In the case of low energetic disorder it can give rise to weakly temperature dependent mobilities, as observed in our systems, and will be discussed in detail in Sec. IV B 2.

Another possible solution resulting in a temperature independent mobility is based on the Holstein polaron model. ${ }^{21,22}$ This model has been applied to discotic and calamitic systems in the past, ${ }^{23-26}$ in order to explain the lack of temperature dependent transport found in many LC mesophases and is applied in Sec. IV B 1.

\section{Holstein small polaron}

In the Holstein model, the charge forms a localized polaron by distorting its surroundings, and this polaron is characterized by its binding energy $E_{p}$ and the transfer integral (bandwidth) between neighboring sites, $J$. In the nonadiabatic limit, that is, when the nearest-neighbor transfer integral $J$ between two hopping sites is much smaller than the polaron binding energy $E_{p}$, i.e., $J \ll E_{p}$. The Holstein polaron mobility can be expressed as

$$
\mu_{p}=\frac{1}{\left(k_{B} T\right)^{3 / 2}} \frac{e d^{2}}{\hbar}\left(\frac{\pi}{2 E_{p}}\right)^{1 / 2} J^{2} \exp \left(\frac{-E_{p}}{2 k_{B} T}\right),
$$

where $d$ is the intermolecular distance and the other symbols have their usual meanings. We notice that Eq. (1) contains both preexponential and exponential factors that depend on $T^{-1}$. It is therefore possible that, given the right combination

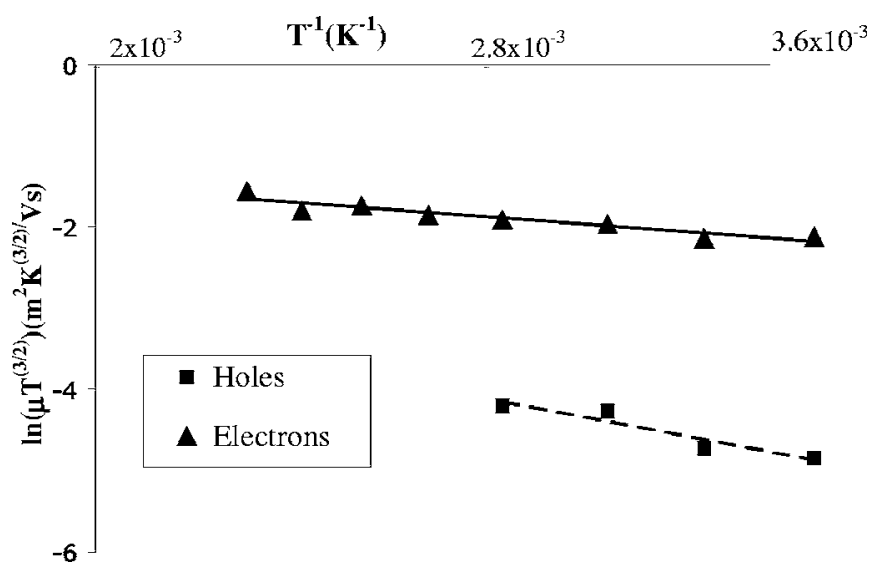

FIG. 16. The data for PTTP-oxetane after polymerization fitted to the small polaron model. $\ln \left(\mu T^{3 / 2}\right)$ vs $T^{-1}$ is plotted and the straight lines are fitted to the data. The deduced parameters are given in Table V.

of binding energy and bandwidth values, an increase in temperature would lead to a drop in the preexponential factor which would compensate the rise due to the exponential factor, and so result in a temperature independent mobility.

Liquid crystalline materials exhibit weak van der Waals intermolecular interactions and because the transfer integral $(J)$ is a measure of the interaction energy between two neighboring molecules, we would expect $J$ to be small, thus justifying the use of the nonadiabatic limit.

Equation (1) implies that a plot of $\ln \left(\mu T^{3 / 2}\right)$ against $T^{-1}$ would give a straight line with a gradient $m$, given by

$$
m=\frac{-E_{p}}{2 k_{B}},
$$

and $y$ axis intercept $C$, given by

$$
C=\ln \left[\frac{1}{\left(k_{B}\right)^{3 / 2}} \frac{e d^{2}}{\hbar}\left(\frac{\pi}{2 E_{p}}\right)^{1 / 2} J^{2}\right] .
$$

Thus $E_{p}$ and $J$ can be recovered from such a plot.

Mobility-temperature data for all three polymerized systems were fitted to Eq. (1). One such example (PTTPoxetane) is shown in Fig. 16 and the resulting values for the transfer integral $J$ and the polaron binding energy for all three systems can be found in Table V. In all cases, a typical smectic phase intermolecular distance $d=4.5 \AA$ was used for the calculations. Although this is a realistic value, we note that any error in the intermolecular distance value used

\begin{tabular}{|c|c|c|c|c|c|c|}
\hline \multirow[b]{2}{*}{ Material } & \multicolumn{2}{|c|}{$\begin{array}{c}\text { Polaron binding } \\
\text { energy }\left(E_{p}\right) \\
(\mathrm{meV})\end{array}$} & \multicolumn{2}{|c|}{$\begin{array}{c}\text { Transfer integral }(J) \\
(\mathrm{meV})\end{array}$} & \multicolumn{2}{|c|}{$\begin{array}{l}\text { Mobility }(\mu) \\
\left(\mathrm{cm}^{2} / \mathrm{V} \mathrm{s}\right)\end{array}$} \\
\hline & Holes & Electrons & Holes & Electrons & Holes & Electrons \\
\hline PTTP-diene & 102 & 85 & 1 & 1 & $7.1 \times 10^{-4}$ & $7.5 \times 10^{-4}$ \\
\hline PTTP-oxetane & 194 & 78 & 19 & 18 & $1.6 \times 10^{-2}$ & $2.8 \times 10^{-2}$ \\
\hline QT-oxetane & 130 & 49 & 3 & 1 & $1.6 \times 10^{-3}$ & $1.1 \times 10^{-3}$ \\
\hline
\end{tabular}
would only affect the calculated transfer integral.

TABLE V. Summary of polaron parameters of polymerized systems. 
With the exception of electrons in PTTP-oxetane, we note that the recovered values in Table V satisfy the condition that the Polaron binding energy $E_{p}$ must be much larger than the transfer integral $J$, i.e., $J \ll E_{p}$, confirming the applicability of the nonadiabatic limit. Although the Holstein small polaron model is strictly one dimensional, it seems to fit the data well in what is expected to be, essentially, two dimensional transport within smectic lamella. All calculated transfer integral values are below $30 \mathrm{meV}$, as expected in the case of organic molecular crystals, ${ }^{27}$ and in agreement with literature values on similar systems. ${ }^{23,26}$ The transfer integral values for both electrons and holes in PTTP-oxetane (18 and $19 \mathrm{meV}$, respectively) are much larger than those calculated for the other systems (ranging from $1 \mathrm{eV}$ to $3 \mathrm{meV}$ ) and in qualitative agreement with the relatively higher mobility measured in this system, as a greater wave function overlap would result in higher charge mobility. Polaron binding energy values derived for holes in the three systems range from 102 to $194 \mathrm{meV}$. These are of the expected order of magnitude for such systems ${ }^{23}$ and compare favorably with those derived for the calamitic smectic system 8PNP-O12. ${ }^{23}$ In all cases, the polaron binding energies calculated for electrons were smaller than those for the corresponding holes, although they are of the same order of magnitude as the hole values, and therefore seem plausible. Because of the prevalence of hole transporting organic materials in the literature, similar analyses have reported hole binding energies and transfer integrals only, ${ }^{23-26}$ making electron value comparisons impossible.

\section{Bässler Gaussian disorder formalism}

In this model the site energies have a Gaussian distribution, width $\sigma$, whereas the positional disorder of the sites is characterized by the dimensionless parameter $\Sigma$. This has previously been applied to calamitic systems. ${ }^{28}$ The empirical expression for the field and temperature dependence of the carrier mobility is given by ${ }^{19}$

$$
\mu(T, E)=\mu_{0} e^{-\left(2 \sigma / 3 k_{B} T\right)^{2}} e^{C_{0} \sqrt{E}\left[\left(\sigma / k_{B} T\right)^{2}-\Sigma^{2}\right]}
$$

in the case of large positional disorder [in the case of small positional disorder, $\Sigma<1.5$; the expression $\Sigma^{2}$ in Eq. (4) is

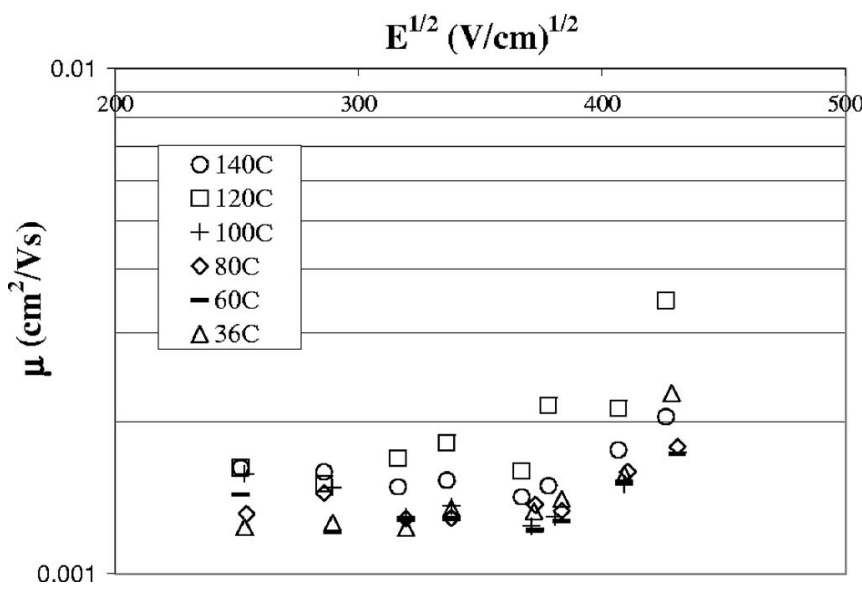

FIG. 17. Poole-Frenkel plot of polymerized QT-oxetane hole mobilities, parametric in temperature.

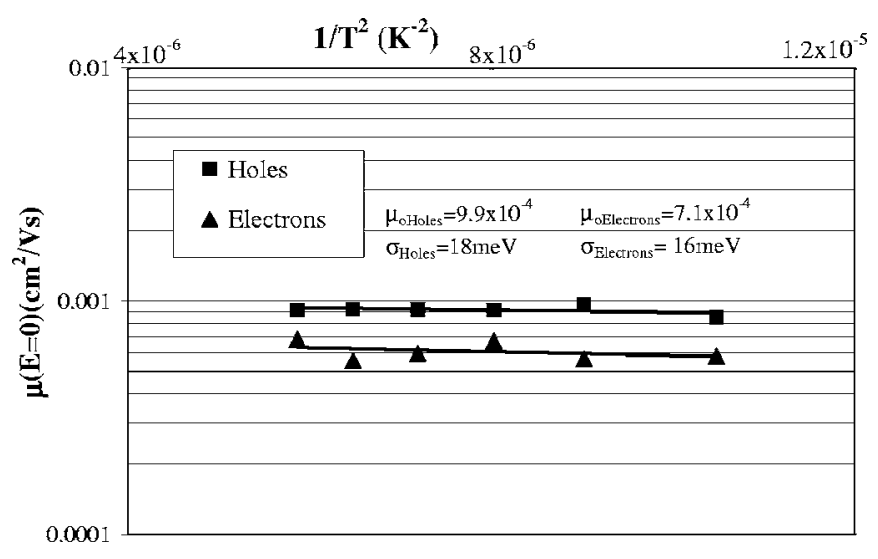

FIG. 18. The zero field mobility vs $1 / T^{2}$ for polymerized QT-oxetane.

replaced by 2.25]. The prefactor $\mu_{0}$ represents the zero field, infinite temperature mobility, whereas the constant $C_{0}$ is empirically determined [it has been determined as 2.9 $\times 10^{-4}(\mathrm{~cm} / \mathrm{V})^{1 / 2}$ for a $0.6 \mathrm{~nm}$ cubic lattice]. A typical Poole-Frenkel plot of mobility, parametric in temperature, is shown in Fig. 17. The superposition of the different temperature data sets clearly demonstrates the lack of temperature dependence in the transport. By fitting exponential expressions to each data set one can extract the zero field mobility as well as the field dependence of the mobility. According to Eq. (4), the zero field mobility should have the following behavior:

$$
\mu(0, T)=\mu_{0} e^{-\left(2 \sigma / 3 k_{B} T\right) 2} .
$$

Thus, by plotting $\ln [\mu(0, T)]$ vs $1 / T^{2}$ one can extract both the prefactor $\mu_{0}$ and the energetic disorder $\sigma$ from the intercept and gradient, respectively. One such plot is shown in Fig. 18; we note that it has a negative gradient, consistent with theory. The derived parameters are summarized in Table VI. We note that in all cases the energetic disorder parameters are much smaller than those found in polymeric systems ( $\sim 20$ compared to $\sim 100 \mathrm{meV}$ ). Small energetic disorder values have previously been reported by Ohno and Hanna $^{28}$ in comparable calamitic systems $(\sim 60 \mathrm{meV}$ in SmB $8 \mathrm{PNPO12}$ ), although we note that in their case those were the values used in Monte Carlo simulations which agreed with experiment, rather than extracting the values from the temperature dependence as presented here. A similar approach to Ohno and Hanna was used by Bleyl et al. ${ }^{29}$ to calculate small energetic disorder values in discotic systems (48 meV in columnar triphenylene dimer H4TD10). One major source of the energetic disorder in polymeric systems is believed to be the variation in conjugation lengths. This is

TABLE VI. Summary of Bässler parameters for polymerized systems.

\begin{tabular}{cccccc}
\hline \hline & \multicolumn{2}{c}{$\sigma(\mathrm{meV})$} & & \multicolumn{2}{c}{$\mu_{0}\left(\mathrm{~cm}^{2} / \mathrm{V} \mathrm{s}\right)$} \\
\cline { 2 - 3 } \cline { 5 - 6 } Material & Holes & Electrons & & Holes & Electrons \\
\hline PTTP-diene & 26 & $\ldots$ & & $4 \times 10^{-4}$ & $4.3 \times 10^{-4}$ \\
PTTP-oxetane & 24 & 27 & & $2.9 \times 10^{-2}$ & $2.1 \times 10^{-2}$ \\
QT-oxetane & 18 & 16 & & $9.9 \times 10^{-4}$ & $7.1 \times 10^{-4}$ \\
\hline \hline
\end{tabular}


entirely consistent with our findings. In the case of polymerized RM systems the carriers are delocalized within the aromatic cores and there is no distribution of delocalization lengths, thus we expect small energetic disorder values. We note that in the case of the $\mu_{0}$ values found in Table VI, only the value obtained for PTTP-oxetane agrees with literature values in other systems $\left(\sim 10^{-2} \mathrm{~cm}^{2} \mathrm{~V}^{-1} \mathrm{~s}^{-1}\right),{ }^{15,16,28,29}$ with the values obtained for the two other systems appearing surprisingly small.

The positional disorder $\Sigma$ and empirical constant $C_{0}$ values are usually extracted from the field and temperature dependence of the mobility by the following method. Rewriting Eq. (4) as

$$
\begin{aligned}
\mu(T, E)= & \mu_{0} e^{-\left(2 \sigma / 3 k_{B} T\right)^{2}} e^{\alpha \sqrt{E}} \\
& \text { where } \alpha=C_{0}\left[\left(\frac{\sigma}{k_{B} T}\right)^{2}-\Sigma^{2}\right]
\end{aligned}
$$

and taking the logarithm of the expression and differentiating with respect to $\sqrt{E}$ yields

$$
\frac{\partial \ln \mu(T, E)}{\partial \sqrt{E}}=\alpha=C_{0}\left(\frac{\sigma}{k_{B} T}\right)^{2}-C_{0} \Sigma^{2} .
$$

A plot of $\alpha$ vs $\left(\sigma / k_{B} T\right)^{2}$ should have a gradient equal to $C_{0}$ and $y$-axis intercept equal to $-C_{0} \Sigma^{2}$. Unfortunately the amount of scatter in all plots of $\alpha$ vs $\left(\sigma / k_{B} T\right)^{2}$ obtained with the polymerized RM results was totally unacceptable, meaning that no values for $\Sigma$ or $C_{0}$ are presented here.

\section{CONCLUSIONS}

Cross-linked networks formed by reactive mesogens form charge transport layers with a wide range of mobilities $\left(10^{-5}-10^{-2} \mathrm{~cm}^{2} \mathrm{~V}^{-1} \mathrm{~s}^{-1}\right)$, offering the choice of unipolar or ambipolar transport and displaying stable, temperature independent mobilities. The range of mobilities available makes them attractive as customized charge transport layers, where balanced hole and electron transport is required for efficient device performance. Although some reactive end groups (e.g., dienes) act as chemical traps for electron transport in reactive mesogens, as indicated by the mobility recovery occurring post-cross-linking, their trapping effect alone is not sufficient to explain the lower mobilities invariably found in RMs, when compared to nonreactive LC systems. The theoretically predicted Holstein small polaron mobility (nonadiabatic limit) adequately explains the weak temperature dependence found in these systems, and the deduced values for the polaron binding energy and transfer integral appear both internally consistent and in agreement with literature values. The weak temperature and field dependence of the mobility in these systems can also be explained in the context of the Bässler Gaussian disorder formalism by the small values obtained for the energetic disorder parameters in agreement with literature. It is worth noting that while the small polaron model only takes into account the microscopic nature of the charge transfer between sites and associated energetic reor- ganization, the energetic disorder model takes into account the transport within a distribution of site energies, neglecting the microscopic nature of the charge transfer. A more complete theoretical description of these systems ought to take into account both phenomena.

\section{ACKNOWLEDGMENTS}

We wish to thank Dr. K. S. Whitehead (Imperial) for the useful discussions on reactive mesogens. Dr. K. J. Donovan (Queen Mary) for help regarding the Holstein polaron model, Professor J. Goodby and Dr. S. Cowling (York) for the identification of the material mesophases, and EPSRC for financial support of this research.

${ }^{1}$ M. Funahashi and J. Hanna, Phys. Rev. Lett. 78, 2184 (1997).

${ }^{2}$ M. Funahashi and J. Hanna, Appl. Phys. Lett. 71, 602 (1997).

${ }^{3}$ D. J. Broer, G. Challa, and G. N. Mol, Macromol. Chem. Phys. 192, 59 (1991).

${ }^{4}$ I. McCulloch, W. Zhang, M. Heeney, C. Bailey, M. Giles, D. Graham, M. Shkunov, D. Sparrowe, and S. Tierney, J. Mater. Chem. 13, 2436 (2003). ${ }^{5}$ M. Shkunov, W. Zhang, D. Graham, D. Sparrowe, M. Heeney, M. Giles, S. Tierney, C. Bailey, I. McCulloch, and T. Kreouzis, Proc. SPIE 5217, 181 (2003).

${ }^{6}$ S. R. Farrar, A. E. A. Contoret, M. O’Neill, J. E. Nicholls, G. J. Richards, and S. M. Kelly, Phys. Rev. B 66, 125107 (2002).

${ }^{7}$ M. Funahashi and J. Hanna, Appl. Phys. Lett. 73, 25 (1998).

${ }^{8}$ M. Mushrush, A. Facchetti, M. Lefenfeld, H. E. Katz, and T. J. Marks, J. Am. Chem. Soc. 125, 9414 (2003).

${ }^{9}$ M. Halik, H. Klauk, U. Zschieschang, G. Schmid, W. Radlik, S. Ponomarenko, S. Kirchmeyer, and W. Weber, J. Appl. Phys. 93, 2977 (2003).

${ }^{10}$ M. Heeney, W. Zhang, S. Tierney, D. Sparrowe, M. Shkunov, and I. McCulloch, U.S. Patent No. 20050184274 (2005).

${ }^{11}$ H. Thiem, P. Strohriegl, M. Shkunov, and I. McCulloch, Macromol. Chem. Phys. 206, 2153 (2005).

${ }^{12}$ P. Vlachos, S. M. Kelly, B. Mansoor, and M. O'Neill, Chem. Commun. (Cambridge) 2002, 874.

${ }^{13}$ M. O'Neil and S. M. Kelly, Adv. Mater. (Weinheim, Ger.) 15, 1135 (2003).

${ }^{14}$ T. Kreouzis, R. J. Baldwin, M. Shkunov, I. McCulloch, M. Heeney, and W. Zhang, Appl. Phys. Lett. 87, 172110 (2005).

${ }^{15}$ M. Funahashi and J. Hanna, Chem. Phys. Lett. 397, 319 (2004).

${ }^{16}$ T. Kreouzis, D. D. C. Bradley, and A. J. Campbell, Proc. SPIE 5214, 141, (2004).

${ }^{17}$ T. Kreouzis, D. Poplavskyy, S. M. Tuladhar, M. Campoy-Quiles, J. Nelson, A. J. Campbell, and D. D. C. Bradley, Phys. Rev. B 73, 235201 (2006).

${ }^{18}$ T. Kreouzis, K. J. Donovan, N. Boden, R. J. Bushby, O. R. Lozman, and Q. Liu, Chem. Phys. 112, 1541 (2000).

${ }^{19}$ H. Bässler, Phys. Status Solidi B 175, 15 (1993).

${ }^{20}$ S. V. Novikov, D. H. Dunlap, V. M. Kenkre, P. E. Parris, and A. V. Vannikov, Phys. Rev. Lett. 81, 4472 (1998).

${ }^{21}$ T. Holstein, Ann. Phys. (N.Y.) 8, 325 (1959).

${ }^{22}$ T. Holstein, Ann. Phys. (N.Y.) 8, 343 (1959).

${ }^{23}$ I. Shiyanovskaya, K. D. Singer, R. J. Twieg, L. Sukomlinova, and V. Gettwert, Phys. Rev. E 65, 041715 (2002).

${ }^{24}$ I. Shiyanovskaya, K. D. Singer, V. Percec, T. K. Bera, Y. Miura, and M. Glodde, Phys. Rev. B 67, 035204 (2003).

${ }^{25}$ T. Kreouzis, K. J. Donovan, N. Boden, R. J. Bushby, O. R. Lozman, and Q. Liu, J. Chem. Phys. 114, 4 (2001).

${ }^{26}$ K. J. Donovan, T. Kreouzis, K. Scott, J. C. Bunning, R. J. Bushby, N. Boden, O. R. Lozman, and B. Movaghar, Mol. Cryst. Liq. Cryst. 396, 91 (2003).

${ }^{27}$ M. Pope and C. E. Swenberg, Electronic Process in Organic Crystals and Polymers (Oxford University Press, Oxford, New York, 1999).

${ }^{28}$ A. Ohno and J. Hanna, Appl. Phys. Lett. 82, 751 (2003).

${ }^{29}$ I. Bleyl, C. Erdelen, H.-W. Schmidt, and D. Haarer, Philos. Mag. B 79, 463 (1999). 